\title{
Written reflection in an eHealth intervention for adults with type 2 diabetes mellitus: a qualitative study
}

This article was published in the following Dove Press journal: Patient Preference and Adherence

\author{
Silje S Lie' \\ Bjørg Karlsen' \\ Christopher P Niemiec ${ }^{2}$ \\ Marit Graue ${ }^{3}$ \\ Bjørg Oftedal' \\ 'Department of Public Health, \\ Faculty of Health Sciences, University \\ of Stavanger, Stavanger, Norway; \\ ${ }^{2}$ Department of Clinical and Social \\ Sciences in Psychology, University \\ of Rochester, Rochester, NY, USA; \\ ${ }^{3}$ Center for Evidence-Based Practice, \\ Western Norway University of \\ Applied Sciences, Bergen, Norway
}

Correspondence: Silje S Lie Department of Public Health, Faculty of Health Sciences, University of Stavanger, N-4036 Stavanger, Norway

Tel +4797506752

Email silje.s.lie@uis.no
Background: Individuals with type 2 diabetes mellitus (T2DM) are responsible for the daily decisions and actions necessary to manage their disease, which makes self-management the cornerstone of diabetes care. Many patients do not reach recommended treatment goals, and thus it is important to develop and evaluate innovative interventions that facilitate optimal motivation for adequate self-management of T2DM.

Objective: The aim of the current study was to explore how adults with T2DM experience using reflection sheets to stimulate written reflection in the context of the Guided Self-Determination (GSD) eHealth intervention and how written reflection might affect their motivation for self-management of T2DM.

Methods: We used a qualitative design in which data were collected through individual interviews. The sample consisted of 10 patients who completed the GSD eHealth intervention, and data were analyzed using qualitative content analysis.

Results: The qualitative content analysis yielded 2 main themes. We labeled the first theme as "Written reflection affects awareness and commitment in diabetes self-management", which reflects 2 subthemes, namely, "Writing creates space and time for autonomous reflection" and "Writing influences individuals' focus in diabetes self-management". We labeled the second theme as "Written reflection is perceived as inapplicable in diabetes self-management", which reflects 2 subthemes, namely, "Responding in writing is difficult" and "The timing of the writing is inappropriate".

Conclusion: Our findings indicate that written reflection in the context of the GSD eHealth intervention may be conducive to motivation for diabetes self-management for some patients. However, it seems that in-person consultation with the diabetes nurse may be necessary to achieve the full potential benefit of the GSD as an eHealth intervention. We advocate further development and examination of the GSD as a "blended" approach, especially for those who consider written reflection to be difficult or unfamiliar.

Keywords: eHealth, guided self-determination, self-determination theory, self-management, type 2 diabetes mellitus, written reflection

\section{Introduction}

Type 2 diabetes mellitus (T2DM) is a chronic health condition whose worldwide prevalence has increased rapidly in recent decades. ${ }^{1}$ Individuals with T2DM are responsible for the daily decisions and actions necessary to manage their disease, which makes self-management the cornerstone of diabetes care. ${ }^{2}$ Self-management can be defined as an "individual's ability to manage the symptoms, treatment, physical and psychosocial consequences and life style changes inherent in living with a chronic condition". 3 
Adequate self-management of T2DM is therefore a complex process that requires motivation for managing medication as well as lifestyle changes in diet and physical activity to reach treatment goals for glycosylated hemoglobin $\left(\mathrm{HbA}_{1 \mathrm{c}}\right)$, cholesterol, and blood pressure in order to prevent serious long-term complications. ${ }^{4,5}$ Indeed, long-term complications associated with T2DM include cardiovascular disease, neuropathy, nephropathy, and periodontal disease, among others. ${ }^{1,5}$ Patients have described adequate self-management of T2DM as difficult to attain because of the following reasons: cumbersomeness of lifestyle changes in diet and physical activity, and the long-term complications of T2DM and other chronic conditions. ${ }^{6}$ Moreover, the values that people hold can conflict with the recommended behaviors for adequate self-management of T2DM, which can undermine the motivation for lifestyle changes. ${ }^{7}$ Hence, it is important to develop and evaluate innovative interventions that facilitate optimal motivation for adequate self-management of T2DM.

Indeed, eHealth interventions have been shown to have potential to support adequate self-management of T2DM, and recommendations suggest that eHealth interventions be theory-based and include "soft-touch" strategies such as personal feedback to enhance efficiency and engagement. ${ }^{8-11}$ Such features enable asynchronous and flexible follow-up for each patient, which can bridge the gap between diabetes care and adequate self-management. Based on these recommendations, in the development phase of our project, we adapted the Guided Self-Determination (GSD) self-management support program to be an eHealth intervention for adults with T2DM. ${ }^{12}$ Originally, the GSD program was developed for type 1 diabetes, and research indicates that the program is effective in facilitating the development of life skills and lowering psychosocial distress. ${ }^{13-20}$

Based on self-determination theory (SDT), the GSD program is intended to enhance autonomous problem solving, goal setting, and action planning among individuals with diabetes. ${ }^{21}$ SDT is an organismic approach to human motivation, which has been applied to health care and health behavior change, including management of T2DM. Central to SDT is the specification of 3 basic psychological needs, namely, autonomy (an experience of volition and choicefulness), competence (an experience of capability and mastery), and relatedness (an experience of support from and connection with important others); the satisfaction of these needs is necessary for optimal motivation, physical health, social integration, and psychological wellness. ${ }^{22-24}$ Indeed, past research has shown that support for the basic psychological needs is associated with higher levels of autonomous motivation for diabetes self-management, medication adherence, quality of life, dietary self-care, and glucose control..$^{4,22,25-28}$

An important feature of the GSD program is the use of semistructured reflection sheets, which are designed to afford patients an opportunity to express their experiences and personal difficulties with diabetes, as well as to enable them to participate actively in their care process. ${ }^{13}$ Such expression and active participation can empower patients to become self-determined and develop the skills necessary for adequate self-management of diabetes. ${ }^{12}$ Written reflection requires the translation of emotions and experiences into words, and this cognitive process can benefit individuals in a variety of situations. ${ }^{29}$ The use of writing as a therapeutic approach has been examined in a variety of populations, including college students who are vulnerable to depression, cancer survivors, and individuals with chronic pain and various physical diseases, and findings indicate that this approach can improve treatment outcomes and quality of life. ${ }^{30-33}$ In addition, a systematic review of interventions for women with breast cancer found that expressive writing can improve their physical health. ${ }^{34}$ To our knowledge, written reflection has not been examined in the context of eHealth interventions, and the current study was designed to fill this gap in the literature.

\section{The study}

\section{Aim}

The aim of the current study was to explore how adults with T2DM experience using reflection sheets to stimulate written reflection in the context of the GSD eHealth intervention, and how written reflection might affect their motivation for self-management of T2DM.

\section{Design}

The current study, which was conducted as a pilot study, is part of a larger project that developed a complex eHealth intervention for adults with T2DM who are treated in general practices in Norway. ${ }^{12}$ We used a qualitative design in which data were collected through individual interviews that were conducted between December 2015 and December 2016. Interviews provide valuable information on patients' experiences and opinions, which is important when piloting clinical interventions in real-life contexts. ${ }^{35}$

\section{Description of the GSD eHealth intervention}

Nurses who were trained in the GSD method and had experience with diabetes care delivered the GSD eHealth intervention to patients in general practices. The GSD eHealth intervention was delivered along with regular care, which 


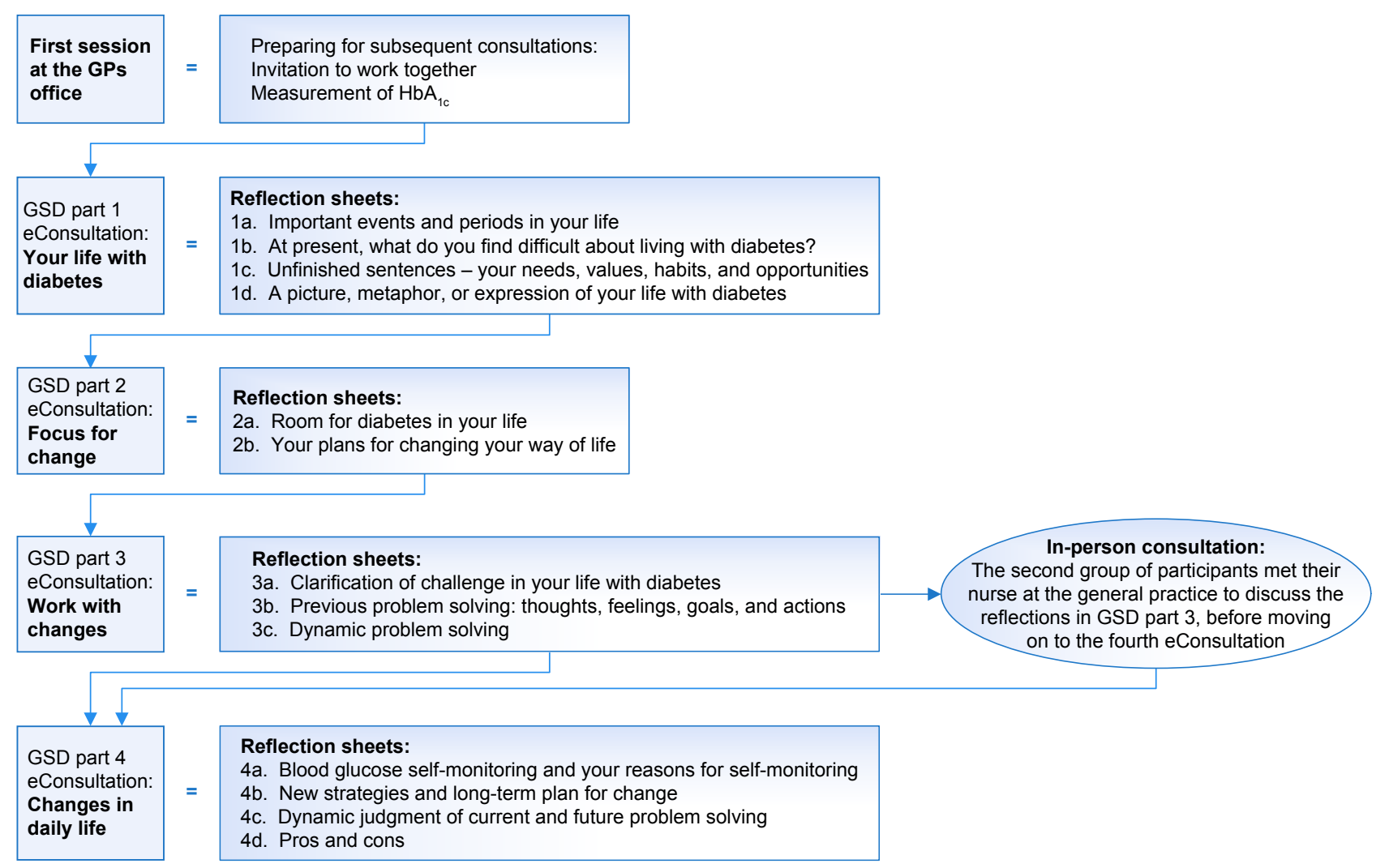

Figure I Overview of the GSD eHealth program for adults with T2DM.

Abbreviations: GP, general practitioner; GSD, Guided Self-Determination; HbA

for individuals with T2DM in Norway consists of structured annual consultations at general practices, regular measurement of $\mathrm{HbA}_{1 \mathrm{c}}$, and additional consultations as per individual needs. ${ }^{5}$ Initially, nurses and participants met face-to-face in order to establish a relationship, during which the nurse explained the aim of the GSD program, how to log on to the Web portal (www.MinJournal.no) and use the secure messaging system, and how to complete the reflection sheets. The Web portal requires electronic identification via BankID, which is aligned with the level of security necessary to allow for transfer of sensitive information in Norway. All participants received a comprehensive manual that described how to use the Web portal. After the initial meeting, participants received the reflection sheets in PDF format via 4 eHealth consultations. They were asked to reflect on and write about their thoughts, feelings, experiences, and difficulties related to the self-management of T2DM, as well as to formulate goals and action plans for adequate self-management of $\mathrm{T} 2 \mathrm{DM}$, and return the completed reflection sheets to the diabetes nurse via secure messages.

The GSD eHealth intervention was initially conducted as a "pure" eHealth intervention by recording responses to the reflection sheets in writing and communicating via secure messages. Due to a long duration (up to 35 weeks) and a large dropout rate, the approach was modified to a "blended" intervention, including 1 in-person consultation with the nurse following the third eHealth consultation. ${ }^{36}$ The participants who were offered an additional in-person meeting completed the intervention in about 12 weeks. Figure 1 presents an overview of the GSD eHealth intervention for T2DM, along with the topics of the 13 reflection sheets and a description of the 1 additional in-person meeting.

\section{Patients and methods Participants and procedure}

At 8 general practices in Norway, participants were recruited by their nurse or general practitioner to participate in the GSD eHealth intervention. Patients were eligible if they had been diagnosed with T2DM for $>3$ months, were at least 18 years of age, could read and communicate in Norwegian, had regular access to the Internet and a computer, and had a registered BankID (a secure personal electronic identification that was necessary to access the Web portal). Patients were excluded if they had severe physical or mental illness that would limit their ability to participate in the study.

A total of 25 patients (18 in the "pure" eHealth intervention, and 7 in the "blended" intervention) from southwestern Norway were invited to participate in the study. Five of the 
18 patients in the "pure" eHealth intervention completed the study, and the large proportion of dropouts in this group has been described elsewhere. ${ }^{36}$ Five of the 7 patients in the "blended" intervention completed the study. Hence, the current study included 10 participants (6 female, 4 male). After completing the intervention, participants were asked by their nurses to take part in an individual interview with an investigator at a time and place of their choosing. All 10 participants agreed to this request. Table 1 presents the characteristics of the study participants.

\section{Data collection}

A semistructured interview guide was used to organize the interviews. Participants were invited to speak freely about the theme addressed in the main question, namely, "What was your overall experience with the GSD eHealth counseling program?" During the conversation, the interviewer asked supplementary questions to clarify and elaborate on participants' responses, including "How did you experience writing your reflections on the digital reflection sheets?" and "How did writing reflections influence your motivation for diabetes self-management?" At the end of each interview, participants were asked to supplement their responses with other experiences related to the GSD eHealth intervention in order to ensure adequate representation of their perspective in the data. On average, interviews took 70 minutes to complete, and all interviews were audiotaped and transcribed

Table I Characteristics of the study participants

\begin{tabular}{|c|c|}
\hline Characteristics & Value \\
\hline \multicolumn{2}{|l|}{ Sex, $n$} \\
\hline Female & 6 \\
\hline Male & 4 \\
\hline Age, mean (range), years & $51(39-64)$ \\
\hline $\mathrm{HbA}_{\mathrm{Ic}}$, mean (range), \% & $7.5(6.0-9.7)$ \\
\hline $\mathrm{BMI}$, mean (range), $\mathrm{kg} / \mathrm{m}^{2}$ & $32(25-39)$ \\
\hline Diabetes duration, median (range) & 4 (3 months- 15 years) \\
\hline \multicolumn{2}{|l|}{ Living situation, $\mathrm{n}$} \\
\hline Alone & I \\
\hline With family & 9 \\
\hline \multicolumn{2}{|l|}{ Educational status, $\mathrm{n}$} \\
\hline Higher education $>4$ years & I \\
\hline Higher education $<4$ years & 4 \\
\hline Upper secondary education & 4 \\
\hline Primary school & l \\
\hline \multicolumn{2}{|l|}{ Occupational status, $\mathrm{n}$} \\
\hline Working full time & 6 \\
\hline Working part time & I \\
\hline Retirement pensioner & I \\
\hline Receiver of disability benefit & I \\
\hline Unemployed & I \\
\hline \multicolumn{2}{|l|}{ Diabetes treatment, $\mathrm{n}$} \\
\hline Diet only & 3 \\
\hline Oral or other medications & 5 \\
\hline Insulin & 2 \\
\hline
\end{tabular}

Abbreviations: $\mathrm{BMI}$, body mass index; $\mathrm{HbA}_{1 \mathrm{c}}$, glycosylated hemoglobin. verbatim. The interviews were conducted in Norwegian. Relevant meaning units were translated into English during the analysis process, and the translation has been text edited. Demographic and clinical data were collected via a questionnaire at baseline.

\section{Ethical considerations}

The Norwegian Regional Committee for Medical and Health Research Ethics (REK West, number 2015/60) approved the study protocol. Prior to the beginning of the study, participants signed a written consent form and were guaranteed anonymity and the right to withdraw from the study at any time. Anonymity was ensured by severing the link between participant names and the ID numbers and transcripts of the interviews.

\section{Data analysis}

We performed a qualitative content analysis, as described by Graneheim and Lundman, ${ }^{37}$ which involved reading in full the unit of analysis (namely, all 10 transcribed interviews). Data from both groups of participants were analyzed together, as the theme focused on experiences with the reflection sheets and writing reflections in the context of the GSD eHealth intervention and how doing so might affect motivation for self-management of T2DM. Meaning units that corresponded to the aim of the study (namely, experiences with using reflection sheets to stimulate written reflection, and how written reflection might affect motivation for self-management of T2DM) were identified and shortened while retaining the main experience, and then labeled with codes. Codes were systematically organized according to their similarities and differences and placed in categories, which describe "what" participants talked about and represent the manifest content of the text. Revision of the codes and the names of categories occurred several times during the process of analysis. Finally, the latent content, or underlying meaning, was interpreted and represented in the subthemes and main themes, which characterize the "'meaningful essence' that runs through the data". ${ }^{38}$ Table 2 presents the themes and subthemes derived

Table 2 Themes and subthemes derived from the qualitative content analysis

\begin{tabular}{ll}
\hline Themes & Subthemes \\
\hline Written reflection affects & Writing creates space and time \\
awareness and commitment & for autonomous reflection \\
in diabetes self-management & Writing influences individuals' \\
& focus in diabetes self-management \\
Written reflection is & Responding in writing is difficult \\
perceived as inapplicable & The timing of the writing is \\
in diabetes self-management & inappropriate \\
\hline
\end{tabular}


from the qualitative content analysis. Abstraction was done in collaboration with coauthors to ensure credibility and to enhance the likelihood that a probable interpretation of the text was obtained.

\section{Findings}

The qualitative content analysis yielded 2 main themes (Table 2) that describe how adults with T2DM experience using reflection sheets to stimulate written reflection in the context of the GSD eHealth intervention and how written reflection might affect their motivation for self-management of T2DM. We labeled the first theme as "Written reflection affects awareness and commitment in diabetes self-management", which reflects 2 subthemes, namely, "Writing creates space and time for autonomous reflection" and "Writing influences individuals' focus in diabetes self-management". We labeled the second theme as "Written reflection is perceived as inapplicable in diabetes self-management", which reflects 2 subthemes, namely, "Responding in writing is difficult" and "The timing of the writing is inappropriate". In the following sections, we describe in detail the content of these themes and subthemes using direct quotations from participants.

\section{Written reflection affects awareness and commitment in diabetes self-management}

Participants suggested that by creating space and time to express thoughts and feelings, writing affords an opportunity for reflection on what is important for them in diabetes self-management. In addition, writing creates transparency and concretizes ideas, which influences focus in diabetes self-management. Hence, written reflection affects awareness and commitment in diabetes self-management.

\section{Writing creates space and time for autonomous reflection}

Participants appreciated the opportunity for reflection in the peace and quiet of their homes, as well as the ability for written reflection without interruption. Participants also valued the opportunity to decide on the timing of their written reflection amid their busy lives, as well as the opportunity to let thoughts "simmer" for a while, which was conducive to mature and thoughtful responses.

I appreciated having the opportunity to sit and relax and fill out [the reflection sheets] in peace and quiet, and to do it when it suited me. That I had time to sit down and prioritize doing it. To sit down and be able to use the time I needed to think through my answers [...]. [Participant 10]
With reflection, participants came to discover aspects of themselves and their reactions to situations of which they had not been aware previously. Participants also appreciated the intellectual stimulation represented by written reflection, through which they could focus on concrete issues and express mature thoughts.

Writing challenges you much more intellectually. That is why writing is very useful. If you just sit and talk, you may put much more emotions into things. When you sit down and write, you dispose some of the emotional, the sentimental, part. You write down your thoughts, cognitive, how you experience the situation. That is why I like to be challenged on that. [Participant 1]

Participants valued the personal nature of written reflection, which afforded an opportunity to think through their responses thoroughly rather than be interrupted with clarifying questions, as typically happens in conversations. Participants considered written reflection to be a useful clinical tool (in addition to traditional health care) because the reflection sheets focused on the psychosocial aspects of having and managing diabetes, and such experiences are important to share with the diabetes nurse.

Earlier follow-up has just been blood samples and other tests, and then finished and "good bye". I have not had time to express thoughts and emotions, and [...] That was what I appreciated, that I could finally communicate with someone about it. How I experience all of it. [Participant 2]

For some participants, written reflection sparked an interest in discussing matters related to self-management of T2DM with their family, which afforded an opportunity for enhanced openness and understanding with important others.

\section{Writing influences individuals' focus in diabetes self-management}

Participants used reflection sheets to create focus in diabetes self-management, as their responses were "in writing". With the opportunity for written reflection, participants created a positive commitment to their goals and action plans, which became specific, concrete, transparent, and manageable and, moreover, could be reviewed after the conclusion of the eHealth consultations.

It becomes more concrete than when it is just in your head. Maybe for some people when they have written it down, I will not say that it becomes a contract, but yet more concrete than when it is just feelings and thoughts. [Participant 4] 
Yet interestingly, some participants expressed the opposite sentiment, such that written reflection can be embellished and/or forgotten after the responses are sent to the diabetes nurse. In response to the Interviewer's question, “Would you go back and check on your goal setting?" 1 participant said, "No, there is no imminent danger of that ever occurring."

\section{Written reflection is perceived as inapplicable in diabetes self-management}

Some participants found it difficult to understand the reflection sheets and respond in writing. Other participants perceived the questions to be repetitive or unnecessary for them. Finally, some participants thought that the timing of the writing was inappropriate, for various reasons. Hence, written reflection is perceived as inapplicable in diabetes self-management.

\section{Responding in writing is difficult}

Some participants mentioned that they struggled with writing in general, whereas others suggested that the writing would have been easier if the reflection sheets were on paper rather than digital. One participant found it difficult to comprehend the questions and, therefore, enlisted family members to help make sense of the reflection sheets. For some of the participants who were offered an in-person meeting following the third eHealth consultation, it was important to discuss the reflection sheets with the diabetes nurse.

I had some problems understanding some of the questions on the reflection sheets. So when I came to see the nurse, I had to say "I don't know what this means", and then she had to explain what it meant. [Participant 7]

Some participants noted the importance of further instruction on how to complete the reflection sheets. Additionally, some participants found the language of the reflection sheets to be "too academic". Other participants found some of the reflection sheets (especially on "Work with changes" [Figure 1]) to be repetitive and difficult to understand/respond to in writing.

But then there were these reflection sheets where I felt like [...] first you were supposed to write about your observations, your thoughts, and feelings. I found those a little hard to separate really. Your observations [...]. What do they mean with that? And then your thoughts and feelings. And then the observations. There you were supposed to write a little without thoughts and feelings? I found this difficult [...]. [Participant 5]

Finally, due to the "locked-to-form" nature of the reflection sheets, some participants perceived less opportunity for elaboration of responses based on individual needs and preferences.

\section{The timing of the writing is inappropriate}

Some participants suggested that the GSD program was introduced either too early or too late in their disease trajectory for them to receive a benefit from written reflection. For some participants, written reflection conflicted with their expectations for a self-management support program. In particular, these participants viewed working with the reflection sheets as too time consuming, likely to create unnecessary problems and concerns, and inapplicable to their current life experience. Other participants focused on personal matters, such as family, relationships, and multimorbidity that undermined their perceived benefit from and opinion of written reflection. They assumed that they were supposed to deal only with specific diabetes self-management behaviors, such as diet and exercise in their written reflections and goal setting. Taken together, the timing of the writing was inappropriate for some participants.

Because you also have other things to deal with. You cannot just put all that aside and simply focus on [diabetes selfmanagement behaviors], right. The other things are there all the time, in the back of my head. [Participant 6]

\section{Discussion}

The aim of the current study was to explore how adults with T2DM experience using reflection sheets to stimulate written reflection in the context of the GSD eHealth intervention and how written reflection might affect their motivation for self-management of T2DM. The findings indicate that participants had diverse experiences with the digital reflection sheets and written reflection more broadly. Some participants experienced written reflection as positively affecting their awareness and commitment in diabetes self-management. On the other hand, some participants experienced difficulties in writing their reflections and perceived this as inapplicable in diabetes self-management. In the following sections, we discuss our findings in the context of previous research and SDT.

\section{Written reflection affects awareness and commitment in diabetes self-management}

One important finding in the current study is that the writing initiated by the digital reflection sheets creates space and time for autonomous reflection, which was experienced as more positive than ordinary follow-up at the general practice. With written reflection, participants were able to 
identify and put into words their personal experiences and difficulties with self-management of T2DM. As the necessary behaviors for self-management of T2DM are demanding and may not have inherent interest for the individual, it is important to support autonomy in health care in order to facilitate optimal, autonomous motivation for diabetes selfmanagement. ${ }^{22,27}$ Individuals experience a sense of autonomy when their behavior is congruent with deeply held values, beliefs, and interests. ${ }^{24}$ Written reflection in the context of the GSD eHealth intervention may be perceived as autonomy supportive, such that it engenders an experience of selfgovernance and volition in patients. These findings build on previous research in which adults with type 1 diabetes perceived their health care climate as more autonomy supportive after participating in the GSD intervention. ${ }^{13}$

Another important finding is that writing influences individuals' focus in diabetes self-management. For some participants, responding to the reflection sheets and then sending these to the diabetes nurse assist in helping to create specific goals and clear action plans, in addition to concretizing what is necessary to attain their goals. The autonomous reflection and the focus created by the writing may have facilitated healthy, autonomous goal setting in the self-management of T2DM. This is important because specific goals are much more effective than general goals for developing effective self-management behaviors., ${ }^{7,39}$ Previous research has shown that active involvement in goal setting is conducive to patients' regulating their self-management behaviors and attaining positive treatment outcomes. ${ }^{23}$ Moreover, competence is supported when individuals pursue goals that they have an opportunity to attain, thereby experiencing a sense of achievement in reaching their goals. ${ }^{24,27}$

Our findings indicate that the GSD eHealth intervention may provide support for patients' competence - as well as autonomy. Indeed, support for competence has been associated with treatment adherence, quality of life, and glycemic control in patients with T2DM. ${ }^{4,26}$ With these findings in mind, we suggest that written reflection in the context of the GSD eHealth intervention may be conducive to positive treatment outcomes because of its potential to support autonomy and competence around self-management of T2DM.

\section{Written reflection is perceived as inapplicable in diabetes self-management}

Our findings also indicate that the GSD eHealth intervention may be described as a "double-edged sword". For some participants, written reflection may affect their awareness and commitment in diabetes self-management in a positive way, whereas for other participants, written reflection was perceived as inapplicable in diabetes self-management. Our findings suggest that responding in writing is difficult and that the timing of the writing is inappropriate for some patients, and thus participants may not value and/or benefit from written reflection in a uniform way. These findings suggest that the reflection sheets might require further adaption for adults with T2DM in an eHealth intervention.

In the current study, the reflection sheets were completed electronically, which contrasts with previous research on the GSD intervention. ${ }^{13,14,18}$ Research on therapeutic writing has shown that the effectiveness of writing as a therapeutic tool depends on support and assistance during the writing process. ${ }^{31}$ Moreover, in previous research showing that the GSD intervention can develop life skills and reduce psychosocial distress in individuals with type 1 diabetes, participants completed the reflection sheets on paper at home as preparation for an in-person consultation with health care personnel, which may facilitate dialogue around assistance with, explanation for, and tailoring of the intervention. ${ }^{13-19}$ The fact that the written reflection and communication with health care personnel occurred primarily electronically may have undermined perceptions of support for some participants.

It is interesting to note that some participants who were offered an in-person meeting following the third eHealth consultation mentioned that their meeting with the diabetes nurse was crucial for understanding the reflection sheets. This finding underscores the importance of in-person consultation that offers assistance to participants around the GSD eHealth intervention and builds on our previous research that revealed participants' missing of in-person consultations with the diabetes nurse as an important contributor to dropping out from the study. ${ }^{36}$ In-person consultation with health care personnel allows for advice based on user reactions to be communicated in real time, which can facilitate engagement in eHealth interventions. Of course, additional in-person consultation can increase the cost and time required for completion of eHealth interventions, in addition to reducing reach into the population. ${ }^{40}$ Nonetheless, we anticipate that the benefits associated with in-person consultation are likely to outweigh the costs.

Some participants considered the timing of the writing to be inappropriate, and thus this aspect of the intervention did not suit them for various reasons. Whereas some participants had a different focus and/or additional challenges in life, others were able to manage their diabetes well without much to consider in written reflection. Hence, it is important to consider the timing of eHealth interventions with regard to disease trajectory, personal needs, and anticipated strains in life. ${ }^{41}$ Furthermore, although - ideally - the reflection 
sheets can be used to consider a broad range of topics in life, participants tend to focus on specific diabetes selfmanagement activities in their goal setting, such as diet and exercise. Future research on written reflection might attempt to strike more of a balance between focusing participants on broad life issues versus specific issues relevant to diabetes self-management.

It is also interesting to consider how the concept of causality orientations within $\mathrm{SDT}^{42}$ might affect perceptions of the timing of the writing as inappropriate. The concept of a causality orientation describes differences in how individuals initiate and regulate their behaviors over extended periods of time, and this concept has received considerable empirical attention. ${ }^{24,43}$ With an autonomy orientation, individuals initiate and regulate their behavior based on personal interest, value, and choice. In contrast, with a controlled orientation, individuals initiate and regulate their behavior based on self- and/or other-imposed perceptions of pressure, coercion, and control. ${ }^{42}$ Certainly, differences in causality orientation might affect the focus of written reflection, the self-management goals that are adopted, and the perception of the GSD eHealth intervention as appropriately timed and beneficial. It is reasonable to speculate that those participants who asserted that written reflection affects awareness and commitment in diabetes self-management (Theme 1) are more likely to have an autonomy causality orientation than those who asserted that written reflection is inapplicable in diabetes self-management (Theme 2). Indeed, individuals who score higher on the controlled causality orientation tend to benefit less from health initiatives such as the GSD program. ${ }^{42}$ Future research on written reflection might examine whether and how the causality orientations affect the amount of benefit that participants derive from the GSD eHealth intervention.

\section{Strengths and limitations}

Several strengths and limitations deserve mention. One strength of the current study was its qualitative design with semistructured interviews during which participants could give voice to their experience with the GSD eHealth intervention. One limitation was the small number of informants $(n=10)$; yet it is important to note that the sample consisted of all participants who completed the GSD eHealth intervention, which precluded the possibility of further recruitment. Indeed, the fact that all participants who completed the intervention agreed to take part in our interviews is a notable strength of the current study. A second strength was that 1 investigator conducted all of the interviews in order to ensure the credibility of the data collection. Undeniably, our findings and interpretations were discussed by all coauthors during analysis and manuscript drafting, which may enhance the trustworthiness of our conclusions. That being said, because a text can have $>1$ meaning and interpretations are subjective, we cannot dismiss the possibility that others would have interpreted our findings in a different way. ${ }^{37,38}$ A second limitation was the heterogeneity in educational status of the study participants, which might have affected how participants responded to the reflection sheets. Half of the participants in the current study had primary or secondary education as their highest level of education. That being said, we found no indication that participants with less education experienced writing as more difficult than those with more education, which may be due to the limits of our small sample size. Thus, it is important for future research with a larger sample size to examine how educational status affects responses to and benefits from written reflection, given the cognitive demands of this component of the eHealth intervention.

\section{Conclusion}

Written reflection stimulated by digital reflection sheets may affect awareness and commitment in diabetes selfmanagement in a positive way by creating space and time for autonomous reflection and influencing individuals' focus in diabetes self-management. Interpreted through the lens of SDT, it is possible that written reflection in the context of the GSD eHealth intervention can support patients' autonomy and competence, which are conducive to autonomous (ie, optimal) motivation for diabetes self-management and positive treatment outcomes. That being said, the structured nature of written reflection in the context of the GSD eHealth intervention may be inapplicable for some participants, as responding in writing can be difficult and the timing of the writing can be inappropriate. Therefore, it seems that in-person consultation with the diabetes nurse may be necessary to achieve the full potential benefit of the GSD as an eHealth intervention. Hence, we advocate for further development and examination of the GSD as a "blended" approach, especially for those who consider written reflection to be difficult or unfamiliar.

\section{Acknowledgments}

The authors express special thanks to the participants involved in the current study. In addition, we express our gratitude to the 8 study nurses and the involved general practices for recruiting the patients and conducting the intervention. 
The current study, which was conducted in collaboration between Western Norway University of Applied Sciences and the University of Stavanger, was funded by a grant from the Norwegian Research Council (project number 221065), and funds from the University of Stavanger and Western Norway University of Applied Sciences, Norway.

\section{Author contributions}

SSL, BK, MG, and BO developed the study design. The interviews, transcriptions, tentative analysis, and first draft of the article were performed by SSL. BK, CPN, MG, and BO were involved in analysis of the data, writing the manuscript, and revising the manuscript for intellectual content. All authors gave final approval of the version to be published and agree to be accountable for all aspects of the work.

\section{Disclosure}

The authors report no conflicts of interest in this work.

\section{References}

1. International Diabetes Federation [webpage on the Internet]. Diabetes Atlas - Eighth Edition; 2017. Available from: https://idf.org/e-library/ welcome.html/. Accessed January 2, 2018.

2. Funnel MM, Anderson RM. Working toward the next generation of diabetes self-management education. Am J Prev Med. 2002; 22(suppl 4):3-5.

3. Barlow J, Wright C, Sheasby J, Turner A, Hainsworth J. Self-management approaches for people with chronic conditions: a review. Patient Educ Couns. 2002;48(2):177-187.

4. Williams GC, Freedman ZR, Deci EL. Supporting autonomy to motivate patients with diabetes for glucose control. Diabetes Care. 1998; 21(10):1644-1651.

5. Helsedirektoratet [webpage on the Internet]. National Guideline Diabetes Treatment. Vol. IS-1674. Oslo: Norwegian Directorate of Health; 2016. Available from: https://helsedirektoratet.no/retningslinjer/ diabetes. Accessed January 13, 2018.

6. Suzuki T, Takei R, Inoguchi T, et al. Clinical significance of barriers to blood glucose control in type 2 diabetes patients with insufficient glycemic control. Patient Prefer Adherence. 2015;9:837-845.

7. Oftedal B, Karlsen B, Bru E. Life values and self-regulation behaviours among adults with type 2 diabetes. J Clin Nurs. 2010;19(17-18): $2548-2556$.

8. Vorderstrasse A, Lewinski A, Melkus GDE, Johnson C. Social support for diabetes self-management via eHealth interventions. Curr Diab Rep. 2016;16(7):1-8.

9. Eland-de Kok P, van Os-Medendorp H, Vergouwe-Meijer A, BruijnzeelKoomen C, Ros W. A systematic review of the effects of e-health on chronically ill patients. J Clin Nurs. 2011;20(21-22):2997-3010.

10. Pal K, Eastwood SV, Michie S, et al. Computer-based diabetes selfmanagement interventions for adults with type 2 diabetes mellitus. Cochrane Database Syst Rev. 2013;(Issue 3):CD008776.

11. Cotter AP, Durant N, Agne AA, Cherrington AL. Internet interventions to support lifestyle modification for diabetes management: a systematic review of the evidence. J Diabetes Complications. 2014;28(2): 243-251.

12. Karlsen B, Oftedal B, Lie SS, et al. Assessment of a web-based Guided Self-Determination intervention for adults with type 2 diabetes in general practice: a study protocol. BMJ Open. 2016;6(12):e013026.
13. Zoffmann V, Lauritzen T. Guided self-determination improves life skills with type 1 diabetes and $\mathrm{A} 1 \mathrm{C}$ in randomized controlled trial. Patient Educ Couns. 2006;64(1-3):78-86.

14. Husted GR, Thorsteinsson B, Esbensen BA, Hommel E, Zoffmann V. Improving glycaemic control and life skills in adolescents with type 1 diabetes: a randomised, controlled intervention study using the Guided Self-Determination-Young method in triads of adolescents, parents and health care providers integrated into routine paediatric outpatient clinics. BMC Pediatr. 2011;11:55.

15. Zoffmann V, Kirkevold M. Realizing empowerment in difficult diabetes care: a Guided Self-Determination intervention. Qual Health Res. 2012;22(1):103-118.

16. Husted GR, Thorsteinsson B, Asbensen BA, et al. Effect of Guided Self-Determination Youth intervention integrated into outpatint visits versus treatment as usual on glycemic control and life skills: a randomized clinical trial in adolescents with type 1 diabetes. Bio Med Central. 2014;15:321.

17. Husted GR, Esbensen BA, Hommel E, Thorsteinsson B, Zoffman V. Adolescents developing life skills for managing type 1 diabetes: a qualitative, realistic evaluation of a Guided Self-Determination-Youth intervention. J Adv Nurs. 2014;70(11):2634-2650.

18. Zoffmann V, Vistisen D, Due-Christensen M. Flexible Guided Self-Determination intervention for younger adults with poorly controlled Type 1 diabetes decreased HbA1c and psychosocial distress in women but not ine men: a real-life RCT. Diabet Med. 2015;32: 1239-1246.

19. Zoffmann V, Prip A, Christiansen AW. Dramatic change in a young woman's perception of her diabetes and remarkable reduction in $\mathrm{HbA} 1 \mathrm{c}$ after an individual course of Guided Self-Determination. BMJ Case Rep. Epub 2015 Jul 6.

20. Mohn J, Graue M, Assmus J, et al. The effect of guided self-determination on self-management in persons with type 1 diabetes mellitus and $\mathrm{HbA} 1 \mathrm{c}$ $\geq 64 \mathrm{mmol} / \mathrm{mol}$ : a group-based randomised controlled trial. BMJ Open. 2017;7(6):e013295.

21. Zoffmann V, Hörnsten Å, Storbækken S, et al. Translating personcentered care into practice: a comparative analysis of motivational interviewing, illness-integration support, and guided self-determination. Patient Educ Couns. 2016;99(3):400.

22. Ryan RM, Deci EL. Self-Determination Theory and the facilitation of intrinsic motivation, social development, and well-being. Am Psychol. 2000;55(1):68-78.

23. Ng JYY, Ntoumanis N, Thøgersen-Ntoumani C, et al. Self-Determination Theory applied to health contexts: a meta-analysis. Perspect Psychol Sci. 2012;7(4):325-340.

24. Niemiec CP, Ryan RM, Deci EL. Self-Determination Theory and the Relation of Autonomy to Self-Regulatory Processes and Personality Development. Oxford, UK: Wiley-Blackwell; 2010.

25. Williams GC, Niemiec CP, Elliot AJ, LaGuardia JG, Gorin AA, Rigby CS. Virtual Look AHEAD Program: initial support for a partly virtualized intensive lifestyle intervention in type 2 diabetes. Diabetes Care. 2014; 37:e169-e170.

26. Williams GC, Patrick H, Niemiec CP, et al. Reducing the health risks of diabetes: how Self-Determination Theory may help improve medication adherence and quality of life. Diabetes Educ. 2009;35(3):484-492.

27. Ryan RM, Patrick H, Deci EL, Williams GC. Facilitating health behaviour change and its maintenance: interventions based on SelfDetermination Theory. Europ Health Psychol. 2008;10:2-5.

28. Austin S, Senécal C, Guay F, Nouwen A. Effects of gender, age, and diabetes duration on dietary self-care in adolescents with type 1 diabetes: a Self-Determination Theory perspective. J Health Psychol. 2011; 16(6):917-928.

29. Pennebaker JW, Chung CK. Expressive writing, emotional upheavals, and health. In: Friedman HS, Silver RC, editors. Foundations of Health Psychology. New York: Oxford University Press; 2007:263-284.

30. Murali C, Fernbach SD, Potocki L. Handing the pen to the patient: reflective writing for children and families affected by genetic conditions. Am J Med Genet A. 2014;164(12):3021-3026. 
31. Furnes B, Dysvik E. Therapeutic writing and chronic pain: experiences of therapeutic writing in a cognitive behavioural programme for people with chronic pain. J Clin Nurs. 2012;21(23-24):3372.

32. Gortner E-M, Rude SS, Pennebaker JW. Benefits of expressive writing in lowering rumination and depressive symptoms. Behav Ther. 2006; 37(3):292-303.

33. Manzoni GM, Castelnuovo G, Molinari E. The WRITTEN-HEART study (expressive writing for heart healing): rationale and design of a randomized controlled clinical trial of expressive writing in coronary patients referred to residential cardiac rehabilitation. Health Qual Life Outcomes. 2011;9:51.

34. Zhou C, Wu Y, An S, Li X. Effect of expressive writing intervention on health outcomes in breast cancer patients: a systematic review and meta-analysis of randomized controlled trials. PLoS One. 2015;10(7): e0131802.

35. Sandelowski M. Using qualitative methods in intervention studies. Res Nurs Health. 1996;19(4):359-364.

36. Lie SS, Karlsen B, Oord ER, Graue M, Oftedal B. Dropout from an eHealth intervention for adults with type 2 diabetes: a qualitative study. J Med Internet Res. 2017;19(5):e187.

37. Graneheim UH, Lundman B. Qualitative content analysis in nursing research: concepts, procedures and measures to achieve trustworthiness. Nurse Educ Today. 2004;24(2):105-112.
38. Graneheim UH, Lindgren B-M, Lundman B. Methodological challenges in qualitative content analysis: a discussion paper. Nurse Educ Today. 2017;56:29-34.

39. Boekaerts M, Niemivirta M. Chapter 13 - self-regulated learning: finding a balance between learning goals and ego-protective goals. In: Boekaerts M, Pintrich PR, Zeidner M, editors. Handbook of SelfRegulation. San Diego: Academic Press; 2000:417-450.

40. Michie S, Yardley L, West R, Patrick K, Greaves F. Developing and evaluating digital interventions to promote behavior change in health and health care: recommendations resulting from an International Workshop. J Med Internet Res. 2017;19(6):e232.

41. Varsi C, Gammon D, Ruland CM, Wibe T. Patients' reported reasons for non-use of an internet-based patient-provider communication service: qualitative interview study. J Med Internet Res. 2013;15(11):e246.

42. Deci EL, Ryan RM. The support of autonomy and the control of behavior. J Pers Soc Psychol. 1987;53(6):1024-1037.

43. Deci EL, Ryan RM. The "What" and "Why" of goal pursuits: human needs and the self-determination of behavior. Psychol Inq. 2000;11(4): $227-268$.

\section{Publish your work in this journal}

Patient Preference and Adherence is an international, peer-reviewed, open access journal that focuses on the growing importance of patient preference and adherence throughout the therapeutic continuum. Patient satisfaction, acceptability, quality of life, compliance, persistence and their role in developing new therapeutic modalities and compounds to optimize clinical outcomes for existing disease states are major areas of interest for the journal. This journal has been accepted for indexing on PubMed Central. The manuscript management system is completely online and includes a very quick and fair peer-review system, which is all easy to use. Visit http://www dovepress.com/testimonials.php to read real quotes from published authors. 